\title{
On the Possibility of Describing the Origin of the Big Bang According to the Hypothesis of Primary Particles
}

\author{
Slobodan Spremo \\ Mathematical Grammar School, Belgrade, Serbia \\ Email: slobodan.spremo@gmail.com
}

How to cite this paper: Spremo, S. (2021) On the Possibility of Describing the Origin of the Big Bang According to the Hypothesis of Primary Particles. Journal of High Energy Physics, Gravitation and Cosmology, 7, 551-558.

https://doi.org/10.4236/jhepgc.2021.72031

Received: March 4, 2021

Accepted: April 18, 2021

Published: April 21, 2021

Copyright $\odot 2021$ by author(s) and Scientific Research Publishing Inc. This work is licensed under the Creative Commons Attribution International License (CC BY 4.0).

http://creativecommons.org/licenses/by/4.0/

\begin{abstract}
In this paper, we have determined the basic physical quantities that describe the very formation of the Big Bang using hypothetical primary particles, in accordance with our Hypothesis of primary particles, as well as with the logically observed smallest increment of speed that can exist, the "speed quantum". According to the Hypothesis of primary particles, they exist in their basic, dynamic state, in their own flat spacetime, moving mutually at speeds much higher than the speed of light in a vacuum. Hence, a certain probability of a collision among these hypothetical particles exists, during which one of them would be abruptly decelerated to a speed greater than the border speed in our Universe, $c$, by a speed quantum, $\varepsilon_{u}$. As shown in this Hypothesis, such deceleration would increase the energy and the momentum of this particle immensely, so that in a very short period of time, they could tunnel into our Universe through the Big Bang, creating our total energy as well as our spacetime. With this theoretical consideration, we determined the power released during the Big Bang itself, the time period during which it took place, and its radius: $P_{B} \approx 1.63 \times 10^{183} \mathrm{~W}, t_{B} \approx 9.51 \times 10^{-114} \mathrm{~s}$ and $r_{B} \approx 2.85 \times 10^{-105} \mathrm{~m}$. Evidently, this approach allowed us to theoretically push the boundaries for the description of this singularity to values lesser than the Planck time and the Planck length. We expect that the results for the initial singularity itself will allow a more detailed study of the Big Bang.
\end{abstract}

\section{Keywords}

Big Bang, Cosmic Microwave Background, Flat spacetime, Planck Mass, Quantum of Speed 


\section{Introduction}

According to our Hypothesis [1], in which we postulated the existence of primary particles that can move at a speed higher than the speed of light in a vacuum, in our flat spacetime we made the conclusion that these particles, at first glance, would have unusual properties. Thus, their energy and their momentum would increase swiftly while slowing down during mutual collisions. We confirmed the possibility of describing the formation of the Big Bang through tunnelling of the acquired energy and momentum of a primary particle through the Big Bang into our Universe, as well as the formation of our energy and matter in our spacetime, utilizing the dynamic properties of these particles described in this way. To be able to test our Hypothesis in a certain way, we were missing the data to what speed, just slightly greater than the border speed $c$, the primary particle could be slowed down to initiate the Big Bang, as well as which amount of basic state energy would be required to be possible to quantitatively describe the Big Bang.

In [2] we saw a logically introduced new term in physics, the "speed quantum". This term allows us to understand that there is a small difference between the actual speed of light in a vacuum and the border speed $c$. For most applications, that minimal difference between these two speeds is not essential, but this "speed quantum" is important to us. This paper uses the apparent fact that we cannot transmit energy to any mass that exceeds the energy of the entire Universe, but also that since the Planck mass $m_{P}$ is associated with the highest level of energy for an accelerated particle, we cannot transfer more energy to any particle than the energy contained in the Planck mass. From these two findings, a "speed quantum" is logically introduced.

\section{Grounds for a Possible Explanation of the Origin of the Big Bang}

\subsection{Hypothesis of Primary Particles}

In this hypothesis, we have postulated primary particles that can move in their own flat spacetime at a speed $u$, that is greater than the speed of light in a vacuum, i.e. $c<u \leq u_{p},\left(u_{p} \gg c\right)$. We have shown that it is possible to extrapolate Einstein's Theory of Special Relativity to these hypothetical particles. We concluded that Einstein's relativity of the notion of simultaneity of some events can be extended to the "world" of primary particles and we determined a $\xi$ factor which corresponds to the Lorentz $\gamma$ factor.

$$
\xi=\frac{1}{\sqrt{1-\frac{c^{2}}{u^{2}}}} .
$$

Also, the identical value of the $\xi$ factor was obtained via transformations of the space-time coordinates for the primary particles. Consequently, we showed that these transformations constitute Lorentz transformations. Based on the $\xi$ 
factor, we introduced the dynamics of primary particles.

These particles would possess fundamental energy

$$
E_{p}=m_{p} c^{2},
$$

in their basic, dynamic state while moving at speeds $u_{p} \gg c$, where $m_{p}$ represents the attributed mass of the primary particle.

In the course of the slowdown of these particles to lesser speeds $u$, during mutual collisions, their total energy $E_{t}$ and momentum $p_{p}$ would increase according to the following equations:

$$
E_{t}=\frac{m_{p} c^{2}}{\sqrt{1-\frac{c^{2}}{u^{2}}}}=\xi m_{p} c^{2},
$$

and

$$
p_{p}=\frac{m_{p} c^{2}}{u \sqrt{1-\frac{c^{2}}{u^{2}}}}=\frac{\xi m_{p} c^{2}}{u},
$$

i.e.

$$
p_{p}=\frac{E_{t}}{u} .
$$

This increase in the total energy of the primary particle during its deceleration logically leads to the introduction of homeokinetic energy $H=E_{t}-E_{p}$, which, in contrast to the kinetic energy, increases with the speed decrement of the primary particle.

\subsection{Determining the Value of the Mass Attributed to the Primary Particle and Its Energy}

Therefore, according to our hypothesis, at the time of the Big Bang, during the mutual collision of two primary particles, one of them could decelerate to a speed just slightly greater than the speed of light $u \rightarrow c$. As we can see from (3), the total energy of the primary particle, and thus its homeokinetic energy, as well as the momentum of the primary particle (4), would increase extremely. The energy and the momentum achieved in such a manner would be tunnelled at that moment through the Big Bang into our Universe, creating our own energy and mass, with the emergence of our spacetime. Therefore,

$$
E_{t}=E_{U},
$$

where $E_{U}=m_{U} c^{2}$ is equivalent to the total mass of the Universe, which according to [2] is $m_{U} \approx 1.73 \times 10^{53} \mathrm{~kg}$. This mass includes every type of mass (baryonic and dark mass) and the mass associated with all types of energy (photons, dark energy, etc.). It is important to note that this would be the mass of the Universe from the perspective of the observer in our position in the Universe, which would differ for the observers depending on their relative movement in relation to us, so e.g. for an observer travelling with a photon that would represent the 
Planck mass. For the Planck mass, we will use:

$m_{P}=\sqrt{\frac{\hbar c}{G}} \approx 1.22 \times 10^{19} \mathrm{GeV} / c^{2} \approx 2.18 \times 10^{-8} \mathrm{~kg}$. Ditto, we will consider the speed of light in a vacuum $c$ a border speed, for according to the Theory of Special Relativity, neither particles nor photons can achieve it, simply because they possess a certain amount of energy. Photons can reach the speed slightly lower than that border speed, i.e., speed which is by a "speed quantum" $\varepsilon_{v} \approx 2.38 \times 10^{-114} \mathrm{~m} \cdot \mathrm{s}^{-1}$ lower than the border speed $c$. This paper, which presented a new cosmological model, among other parameters of the Universe, gave us the apparent mass $m_{U}$ and "speed quantum" $\varepsilon_{v}$, calculated for metrological purposes, which we took for they were logical and compatible with our Hypothesis, allowing us to determine the attributed mass of the primary particle, i.e., its energy.

Thus, according to [2], an observer traveling at a photon speed, by a quantum smaller than the border speed $c$, i.e. $c-\varepsilon_{v}$, would register that the Universe had the Planck mass:

$$
m_{P}=m_{U} \sqrt{1-\frac{\left(c-\varepsilon_{v}\right)^{2}}{c^{2}}} .
$$

As we have shown in [3], from (3) and (6) we can write for the very moment of the Big Bang, where $u \rightarrow c$ :

$$
\frac{m_{p} c^{2}}{\sqrt{1-\frac{c^{2}}{u^{2}}}}=m_{U} c^{2}
$$

so knowing that $u=c+\varepsilon_{u}$, we have

$$
m_{p}=m_{U} \sqrt{1-\frac{c^{2}}{\left(c+\varepsilon_{u}\right)^{2}}}
$$

where $\varepsilon_{u}$ represents the speed quantum of the primary particle.

Further, from (7) and (9) we get:

$$
\frac{m_{p}}{\sqrt{1-\frac{c^{2}}{\left(c+\varepsilon_{u}\right)^{2}}}}=\frac{m_{P}}{\sqrt{1-\frac{\left(c-\varepsilon_{v}\right)^{2}}{c^{2}}}}=m_{U},
$$

and with the logical assumption that $\left(\varepsilon_{u} \ll c\right)$, we can conclude that the attributed mass of the primary particle is equal to the Planck mass

$$
m_{p}=m_{P} \approx 2.18 \times 10^{-8} \mathrm{~kg}
$$

that is, according to (2) its energy is

$$
E_{p} \approx 1.22 \times 10^{19} \mathrm{GeV} .
$$

Now, from (10) we can determine the speed quantum of the primary particle as

$$
\varepsilon_{u}=\frac{c \varepsilon_{v}}{c+\varepsilon_{v}} \approx 2.38 \times 10^{-114} \mathrm{~m} \cdot \mathrm{s}^{-1}
$$


and as we see, the speed quantum of the primary particle $\varepsilon_{u}$ is approximately equal to the speed quantum $\varepsilon_{v}$.

The mass attributed to the primary particle can also be determined from the fact that, in accordance with our hypothesis, the momentum acquired during the deceleration of this particle is tunnelled into the Universe. Considering (4), we have:

$$
m_{p}=\frac{p_{p} u}{c^{2}} \sqrt{1-\frac{c^{2}}{u^{2}}}
$$

In accordance with the law of momentum conservation, the primary particle transmitted its entire momentum into the Universe during the Big Bang, i.e.

$$
p_{p}=p_{U}
$$

where the following applies $p_{U}=m_{U} c$; hence, we get

$$
m_{p}=\frac{m_{U} c\left(c+\varepsilon_{u}\right)}{c^{2}} \sqrt{1-\frac{c^{2}}{\left(c+\varepsilon_{u}\right)^{2}}}=m_{P} .
$$

Therefore, in this way, we also concluded that the attributed mass of the primary particle is equal to the Planck mass.

\section{Description of the Origin of the Big Bang According to the Hypothesis of Primary Particles}

Primary particles in their basic, dynamic state, in their own flat spacetime, can, with some probability, suffer mutual collisions. During such collisions, a possibility would occur that the speed of one of these particles would decrease so much, to cause its energy and momentum to increase extremely, in accordance with (3) and (4). Thus, it is possible that during one of these collisions among the primary particles, the speed of one of them decreases to a value very close to border speed $c$ for the Universe, that is, to speed $c+\varepsilon_{u}$. At that moment, its enormous energy and momentum would be tunnelled into our Universe through a blistering singularity, the Big Bang. The energy tunnelled through the Big Bang into the Universe, according to (8) would equal

$$
E_{U}=\frac{m_{p} c^{2}}{\sqrt{1-\frac{c^{2}}{\left(c+\varepsilon_{u}\right)^{2}}}}=m_{U} c^{2} \approx 1.55 \times 10^{70} \mathrm{~J} .
$$

Figure 1 shows how a sharp speed decrease of the primary particle (blue curve) to a speed by a $\varepsilon_{u}$ greater than the border speed $c$, results in a Big Bang in the area $a$, which displays as magnified. Following the tunnelling of the primary particle energy, our spacetime and energy $E_{U}$ are created (red dot).

The power released during the Big Bang can be determined from (3) as

$$
P_{B}=\frac{\mathrm{d} E_{t}}{\mathrm{~d} t}=-\frac{m_{p} c^{4}}{2\left(u^{2}-c^{2}\right)^{\frac{3}{2}}},
$$




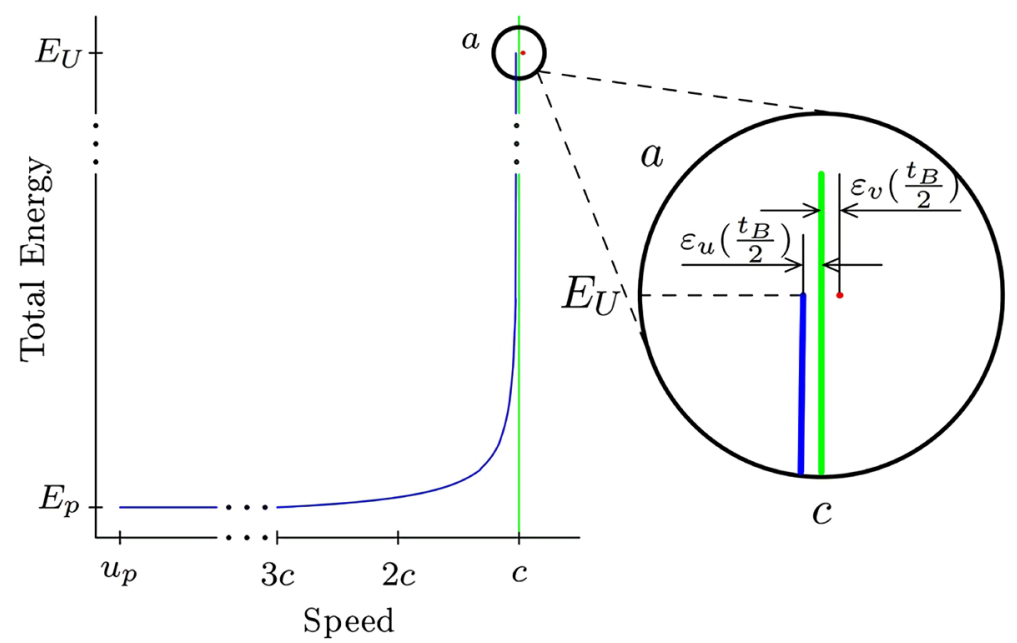

Figure 1. The blue curve represents the energy increase of the primary particle during a sharp decrease in its speed due to a certain collision, to a speed by a quantum $\varepsilon_{u}$ greater than the border speed $c$ (green line), while the red dot represents the creation of total energy of the universe $E_{U}$, resulting from tunnelling of the energy reached by the primary particle. The Big Bang creation area $a$ is shown enlarged as well, showing that the energy of the primary particle is tunnelled into the energy of the universe during $t_{B}$.

where knowing that $u=c+\varepsilon_{u}$, so we get that during the Big Bang, the power of the following intensity was released

$$
P_{B}=\frac{m_{p} c^{4}}{2\left(\left(c+\varepsilon_{u}\right)^{2}-c^{2}\right)^{\frac{3}{2}}} \approx \frac{m_{p} c^{4}}{2\left(2 c \varepsilon_{u}\right)^{\frac{3}{2}}} \approx 1.63 \times 10^{183} \mathrm{~W} .
$$

Thence, the intensity of the force released during the Big Bang would equal

$$
F_{B}=\frac{P_{B}}{C} \approx 5.44 \times 10^{174} \mathrm{~N} .
$$

Knowing that at this power the total energy of the Universe is released (17), we can determine the duration of the Big Bang

$$
t_{B}=\frac{E_{U}}{P_{B}} \approx 9.51 \times 10^{-114} \mathrm{~s} .
$$

Further, the radius of the Big Bang itself would equal

$$
r_{B}=c t_{B} \approx 2.85 \times 10^{-105} \mathrm{~m} \text {. }
$$

Ditto, by utilizing (4), from Newton's second law, we can alternatively determine the force released during the Big Bang

$$
F_{B}=\frac{\mathrm{d} p_{p}}{\mathrm{~d} t}=-\frac{m_{p} c^{2} u \frac{\mathrm{d} u}{\mathrm{~d} t}}{\left(u^{2}-c^{2}\right)^{\frac{3}{2}}},
$$

whence for $u=c+\varepsilon_{u}$, as well as for the minimal alteration of speed $\mathrm{d} u=\varepsilon_{u}$, which occurred during the shortest moment of time $\mathrm{d} t=t_{B} / 2$, during which this change of the speed of the primary particle induced this change in the mo- 
mentum, we obtain that the intensity of the force at the Big Bang has a value of

$$
F_{B}=\frac{\mathrm{d} p_{p}}{\mathrm{~d} t}=\frac{m_{p} c^{2}\left(c+\varepsilon_{u}\right) \frac{\mathrm{d} u}{\mathrm{~d} t}}{\left(\left(c+\varepsilon_{u}\right)^{2}-c^{2}\right)^{\frac{3}{2}}} \approx \frac{m_{p} c^{3} \frac{\varepsilon_{u}}{\frac{1}{2} t_{B}}}{\left(2 c \varepsilon_{u}\right)^{\frac{3}{2}}} \approx 5.44 \times 10^{174} \mathrm{~N} .
$$

In this way, likewise, utilizing (24), we obtain identical values of physical quantities that describe the Big Bang itself, as well as by using (19).

The sign "-" in Equations (18) and (23), results in a fact that the physical quantities that describe the Big Bang itself: power $P_{B}$, force $F_{B}$, time $t_{B}$ and the radius of the Big Bang itself $r_{B}$, also carry the sign “-”, which indicates that these physical quantities offer a physical description, up to the creation of energy $E_{U}$ (red dot in the picture), i.e., up to the creation of our spacetime. However, our spacetime, i.e., our Universe, was created with the creation of energy $E_{U}$, hence, for that very moment, these physical quantities would have a positive sign.

\section{Results and Discussion}

Based on the predicted elementary dynamic properties of the primary particles, which they would possess according to the Hypothesis of primary particles, as well as the calculated energy and momentum of these hypothetical particles and their speed quantum, we quantitatively determined the important physical properties of the Big Bang. These include the force intensity during the Big Bang, the power released during the Big Bang, the period of its duration, as well as the radius within the Big Bang itself generated. Utilizing these quantities, we determined identical values, using both the expression for total energy and the expression for the momentum of the primary particle. The obtained, theoretically logical values for these physical quantities can contribute to further study of the Big Bang, for, as evident, these quantities describe the Big Bang itself deeper than the level which the Theory of Relativity can reach (limited by the Planck time and the Planck length while describing this singularity). Thus, these derived physical quantities allow for the deepest pushing of boundaries in physics, while describing the Big Bang. The results obtained in this paper could be important for the description of the very early Universe, directly after the occurrence of the Big Bang, which we have described in accordance with our hypothesis. Compared to the observational results that we possess currently and will have at our disposal in the future, such a description would also enable the evaluation of our hypothesis, followed by the opening of new horizons in physics.

The most significant observational fact, cosmic microwave background radiation, represents the evidence that our Universe has expanded from a very hot, dense state, which is in line with our results.

We expect this indirect experimental evidence of a new possibility of describing the origin of the Big Bang to be possible in the continuation of future scientific work in this direction, so that we could make an important shift in physics, 
as well as our philosophical view of the world.

\section{Conflicts of Interest}

The author declares no conflicts of interest regarding the publication of this paper.

\section{References}

[1] Spremo, S. (2019) Hypothesis of Primary Particles and the Creation of the Big Bang and Other Universes. Journal of Modern Physics, 10, 1532-1547.

https://doi.org/10.4236/jmp.2019.1013102

[2] Mercier, C. (2019) Calculation of the Mass of the Universe, the Radius of the Universe, the Age of the Universe and the Quantum of Speed. Journal of Modern Physics, 10, 980-1001. https://doi.org/10.4236/jmp.2019.108065

[3] Spremo, S. (2021) Determination of the Energy of a Primary Particle in Accordance with the Hypothesis of Primary Particles and Another Meaning of Planck Mass. Journal of High Energy Physics, Gravitation and Cosmology, 7, 144-148.

https://doi.org/10.4236/jhepgc.2021.71007 